\title{
CLOSED OPERATORS AND EXISTENCE THEOREMS IN MULTIDIMENSIONAL PROBLEMS OF THE CALCULUS OF VARIATIONS ${ }^{1}$
}

\author{
BY L. CESARI AND P. KAISER
}

Communicated by James Serrin, July 13, 1973

1. Weakly and strongly closed operators. Let $X$ be a topological space with topology $\tau$ (say, a Banach space with weak topology), let $S$ be a subset of $X$, and $Y$ a Banach space. An operator $\mathscr{A}: S \rightarrow Y$ (not necessarily linear) is said to be weakly [strongly] closed with respect to $(X, \tau)$ (briefly, $\mathscr{A}$ has property $\mathrm{K}_{w}\left[\mathrm{~K}_{\sigma}\right]$ ) provided $x_{k} \in S, k=1,2, \cdots$, $x \in X, y \in Y, x_{k} \rightarrow x$ in $(X, \tau), \mathscr{A} x_{k} \rightarrow y$ weakly [strongly] in $Y$ implies $x \in S, y=\mathscr{A} x$. Analogously, $\mathscr{A}: S \rightarrow Y$ is said to have the weak [strong] convergence property with respect to $(X, \tau)$ (briefly, $\mathscr{A}$ has property $\left.\mathrm{V}_{w}\left[\mathrm{~V}_{\sigma}\right]\right)$ provided $x_{k} \in S, k=1,2, \cdots, x \in S, x_{k} \rightarrow x$ in $(X, \tau)$ implies $\mathscr{A} x_{k} \rightarrow \mathscr{A} x$ weakly [strongly] in $Y$ as $k \rightarrow \infty$.

Convergence properties $\mathrm{V}_{w}$ and $\mathrm{V}_{\sigma}$ have been already used in [1], [8] toward existence theorems in multidimensional problems of optimization. The closure properties $\mathrm{K}_{w}$ and $\mathrm{K}_{\sigma}$ are used here in this context for the first time. These closure properties (closed graph properties) are well known. (See, e.g., N. Dunford and J. T. Schwartz [11] for linear operators; for nonlinear monotone operators see, e.g., G. J. Minty [14].)

2. Multidimensional problems of optimization with state equations in the strong form. We are interested here in problems of optimization (Lagrange problems, or problems of optimal control) in a fixed bounded domain $G \subseteq E^{v}$. The unknown is an element of a Banach space $X$ with norm $\|x\|$. State equations - in either strong or weak forms-and unilateral constraints are expressed in terms of general not necessarily linear operators on some subsets $S$ of $X$, mapping $S$ into vector-valued $L$-integrable functions on $G$ and $\partial G$ respectively, and of arbitrary measurable vectorvalued control functions $u$ on $G$ and $v$ on $\partial G$.

Precisely, we denote by $\Gamma$ a closed subset of $\partial G$, by $\mu$ a suitable measure function on $\Gamma$, by $T$ the set of all measurable vector functions $u(t)=$ $\left(u^{1}, \cdots, u^{m}\right), t \in G$ (distributed controls), and by $\stackrel{\circ}{T}$ the set of all $\mu$ measurable vector functions $v(t)=\left(v^{1}, \cdots, v^{m^{\prime}}\right), t \in \Gamma$ (boundary controls). We denote by $\mathscr{L}, \mathscr{J}, \mathscr{M}, \mathscr{K}$ given operators $\mathscr{L}: S \rightarrow\left(L_{p}(G)\right)^{r}$,

AMS (MOS) subject classifications (1970). Primary 49A25, 49A50; Secondary $46 \mathrm{E} 35$.

${ }^{1}$ This research was partially supported by AFOSR Research Project 71-2122 at the University of Michigan. 
$\mathscr{J}: S \rightarrow\left(L_{p}(\Gamma)\right)^{r^{\prime}}, \mathscr{M}: S \rightarrow\left(L_{p}(G)\right)^{s}, \mathscr{K}: S \rightarrow\left(L_{p}(\Gamma)\right)^{s^{\prime}}$, where $p \geqq 1$, and $r, r^{\prime}, s, s^{\prime}$ are integers. The images under $\mathscr{L}, \mathscr{J}, \mathscr{M}, \mathscr{K}$ of elements $x \in S$ will be denoted by $z, \stackrel{\dot{z}}{, y}, \dot{y}$, or $z(t)=\left(z^{1}, \cdots, z^{r}\right)=(\mathscr{L} x)(t)$, $t \in G, \stackrel{\circ}{z}(t)=\left(\stackrel{o}{z}^{1}, \cdots, \stackrel{s^{r}}{ }{ }^{\prime}\right)=(\mathscr{J} x)(t), t \in \Gamma ; y(t)=\left(y^{1}, \cdots, y^{s}\right)=(\mathscr{M} x)(t)$, $t \in G, \dot{y}(t)=\left(\dot{y}^{1}, \cdots, \dot{y}^{s^{\prime}}\right)=(\mathscr{K} x)(t), t \in \Gamma$.

We consider the problem of finding elements $x \in S, u \in T, v \in \stackrel{T}{T}$, so as to minimize a cost functional of the form

(1) $I[x, u, v]=\int_{G} f_{\circ}(t,(\mathscr{M} x)(t), u(t)) d t+\int_{\Gamma} g_{\circ}(t,(\mathscr{K} x)(t), v(t)) d \mu$, subject to state equations (strong form)

$$
\begin{array}{ll}
(\mathscr{L} x)(t)=f(t,(\mathscr{M} x)(t), u(t)) & \text { a.e. in } G, \\
(\mathscr{J} x)(t)=g(t,(\mathscr{K} x)(t), v(t)) & \mu \text {-a.e. on } \Gamma,
\end{array}
$$

and unilateral constraints on the values of $u, v, y, \dot{y}$ of the forms

$$
\begin{aligned}
u(t) & \in U(t,(\mathscr{M} x)(t)) \subseteq E^{m} & & \text { a.e. in } G, \\
v(t) & \in V(t,(\mathscr{K} x)(t)) \subseteq E^{m^{\prime}} & & \mu \text {-a.e. on } \Gamma, \\
(\mathscr{M} x)(t) & \in A(t) \subseteq E^{s} & & \text { a.e. in } G, \\
(\mathscr{K} x)(t) & \in B(t) \subseteq E^{s^{\prime}} & & \mu \text {-a.e. on } \Gamma .
\end{aligned}
$$

Here we assume that for any $t \in \operatorname{cl} G$ a given subset $A(t)$ of $E^{s}$ is assigned, and we denote by $A$ the set of all $(t, y) \in E^{v+s}$ with $t \in \operatorname{cl} G, y \in A(t)$. We assume that for any $(t, y) \in A$ a given subset $U(t, y)$ of $E^{m}$ is assigned (distributed control space), and we denote by $M$ the set of all $(t, y, u)$ with $(t, y) \in A, u \in U(t, y)$. Then, $f_{0}(t, y, u), f(t, y, u)=\left(f_{1}, \cdots, f_{r}\right)$ are given functions on $M$. Analogously, we assume that for any $t \in \Gamma$ a given subset $B(t)$ of $E^{s^{\prime}}$ is assigned, and we denote by $B$ the set of all $(t, \stackrel{j}{y}) \in E^{v+s^{\prime}}$ with $t \in \Gamma, \dot{y} \in B(t)$. We assume that for any $(t, \dot{y}) \in B$ a given subset ${ }_{0} V(t, \stackrel{\circ}{y})$ of $E^{m^{\prime}}$ is assigned (boundary control space), and we denote by $\dot{M}$ the set of all $(t, \dot{y}, v)$ with $(t, \dot{y}) \in B, v \in V(t, \dot{y})$. Then, $g_{\circ}(t, \dot{y}, v), g(t, \dot{y}, v)=\left(g_{1}, \cdots, g_{r}\right)$ are given functions on $\stackrel{\circ}{M}$.

We denote by $y=\left(y^{1}, \cdots, y^{8}\right)$ and $y=\left(y^{1}, \cdots, y^{8^{\prime}}\right)$ the state variables of the problem. We denote by $|E|$ the Lebesgue measure of a measurable subset $E$ of $G$, as well as the $\mu$-measure of a $\mu$-measurable subset $E$ of $\Gamma$.

Usually, $X$ is a Sobolev space on $G$, state equation (2) represents a system of $r$ partial differential equations in $G$, and state equation (3) represents either boundary data, or a system of $\boldsymbol{r}^{\prime}$ partial differential equations on $\Gamma$ (or on $\partial G$ ). But the situation may be quite different, since the operators $\mathscr{L}, \mathscr{J}, \mathscr{M}, \mathscr{K}$ need not be differential operators. All we shall require is a set of axioms $(\mathrm{H})$ in the present setting, as well as in the setting of $\S 3$ with state equations in the weak form. In all cases the existence theorems follow in a natural way from uniformly proved 
closure theorems and lower closure theorems, extending the usual lower semicontinuity theorems for classical free problems of the calculus of variations.

$\left(\mathrm{H}_{1}\right)$ Hypotheses on $\mathscr{L}, \mathscr{J}, \mathscr{M}, \mathscr{K}$. We assume that $\mathscr{L}: S \rightarrow\left(L_{1}(G)\right)^{r}$ and $\mathscr{J}: S \rightarrow\left(L_{1}(\Gamma)\right)^{r^{\prime}}$ are weakly closed (property $\mathrm{K}_{w}$ ) and that $\mathscr{M}: S \rightarrow$ $\left(L_{1}(G)\right)^{s}, \mathscr{K}: S \rightarrow\left(L_{1}(\Gamma)\right)^{s^{\prime}}$ have the strong convergence property (property $\left.\mathrm{V}_{\sigma}\right)$ with respect to $(X, \tau)$.

$\left(\mathrm{H}_{2}\right)$ Hypotheses on the set $\Gamma$ and measure $\mu$. We assume that the closed set $\Gamma \subseteq \partial G$ is the union of finitely many sets $\Gamma_{1}, \cdots, \Gamma_{N}$, each $\Gamma_{j}$ being the image of a $(\nu-1)$-dimensional interval $I$ under a transformation $T_{j}$ of class $K$ (Morrey), say $T_{j}: I \rightarrow \Gamma_{j}$. To simplify the exposition we assume that $\mu$ is the hyperarea measure defined on $\Gamma$ by the mappings $T_{j}$.

(C) Property C. Instead of the usual closure and continuity requirements, we shall assume that only the following Carathéodory property (C) holds on $G, A, M, f_{\circ}$ and $f$ : Given $\varepsilon>0$ there is a compact set $C \subseteq G$ with $|G-C|<\varepsilon$ such that the sets $A_{C}=[(t, x) \in A \mid t \in C]$ and $M_{C}=$ $[(t, y, u) \in M \mid t \in C]$ are closed, and $f_{\circ}(t, y, u), f(t, y, u)$ are continuous on $M_{C}$.

For instance, if $A$ and $M$ are closed, and $f_{\circ}(t, y, u), f(t, y, u)$ are measurable in $t$ for every $(y, u)$, and continuous in $(y, u)$ for every $t$, then it is well known that property (C) holds for $A, M, f_{\circ}, f$.

( $\Psi$ ) Growth hypotheses on $f_{\circ}, f$ and $g_{\circ}, g$. We assume that $f_{\circ}$ and $f$ satisfy the following growth property $\left(\Psi^{*}\right)$ : Given $\varepsilon>0$ there is a function $\Psi_{\varepsilon}(t) \geqq 0, t \in G, \Psi_{\varepsilon} \in L_{1}(G)$, such that $|f(t, y, u)| \leqq \Psi_{\varepsilon}(t)+\varepsilon f_{0}(t, y, u)$ for all $(t, y, u) \in M$. Analogous hypotheses (C) and ( $\Psi)$ are made on $\Gamma, B$, $\stackrel{M}{M}, g_{\circ}$ and $g$. Note that property $(\Psi)$ on $f_{\circ}, f$ for $\varepsilon=1$ implies $f_{\circ} \geqq-\Psi_{1}(t)$ with $\Psi_{1} \in L_{1}(G)$, and the analogous property $\left(\Psi^{\prime}\right)$ on $g_{\circ}, g$ implies $g_{\circ} \geqq$ $-\stackrel{\circ}{\Psi}_{1}(t)$ with $\stackrel{\circ}{\Psi}_{1} \in L_{1}(\Gamma)$. Thus, under such properties $(\Psi)$ both integrals in (1), say $I_{1}$ and $I_{2}$ are bounded below, and so is $I[x, u, v]$.

The sets $Q(t, y), R\left(t, y^{\circ}\right)$ and seminormality conditions. For every $(t, y) \in A$ we denote by $\widetilde{Q}(t, y)$ the set of all $(\stackrel{\circ}{z}, z) \in E^{r+1}$ with $i \geqq$ $f_{\circ}(t, y, u), z \underset{\approx}{\approx} f(t, y, u), u \in U(t, y)$. Analogously, for every $(t, y) \in B$ we denote by $\widetilde{R}(t, \dot{y})$ the set of all $(\stackrel{\circ}{z}, z) \in E^{r^{\prime}+1}$ with $z^{\circ} \geqq g_{\circ}(t, \dot{y}, v)$, $z=g(t, y, v), v \in V(t, \dot{y})$. We shall need the convexity of these sets $\widetilde{Q}(t, y)$, $\widetilde{R}(t, y)$, together with some regularity (seminormality) conditions, namely, property (Q) ([1]-[5]), a variant of Kuratowski's upper semicontinuity, or property $(\mathrm{K})[13]$. In [5] Cesari showed that it is enough to require property (Q) of the sets $\widetilde{Q}(t, y), \widetilde{R}(t, \dot{y})$ with respect to the state variables only, $y$ and $\dot{y}$ respectively. Thus, $\widetilde{Q}(t, y)$ is said to have property $(\mathrm{K})$ [(Q)] with respect to $y$ at $(\bar{t}, \bar{y}) \in A$, provided $\widetilde{Q}(\bar{t}, \bar{y})=\bigcap_{\delta} \operatorname{cl} S(\bar{t}, \bar{y}, \delta)$ $\left[\widetilde{Q}(\bar{t}, \bar{y})=\bigcap_{\delta} \operatorname{cl} \operatorname{co} S(\bar{t}, \bar{y}, \delta)\right]$, where $S(\bar{t}, \bar{y}, \delta)$ is the union of all sets $\widetilde{Q}(\bar{t}, y)$ with $y \in A(\bar{t}),|y-\bar{y}| \leqq \delta, \delta>0$, and where cl and co denote 
as usual closure and convex hull. Analogous definitions hold for the sets $\widetilde{R}(t, \stackrel{y}{y})$. Finally, properties $\left(\mathrm{Q}_{\rho}\right)$, intermediate between properties $(\mathrm{Q})$ and (K), have been introduced in [8], and P. Kaiser [12] has shown that these properties too need only be verified with respect to the state variables.

Recently, Cesari has shown [6], [7] that these requirements can be replaced by a different set of hypotheses. For instance, if the sets $\widetilde{Q}(t, y)$ are closed and convex, it is enough to know that (a1) there is a bounded measurable function $p: G \rightarrow E^{r}$, with $p(t) \in Q(t, y)=f(t, y, U(t, y)) \subset E^{r}$ for all $y \in A(t), t \in G$; (a2) for every $N>\sigma=\operatorname{Sup}|p(t)|$, the nonempty convex sets $\widetilde{Q}(t, y) \cap\left[E^{1} \times D(0, N)\right]$ satisfy property $(\mathrm{K})$ with respect to $y$, where $D(0, N)=\left[z \in E^{r}|| z \mid \leqq N\right]$; (a3) for some constant $c \geqq 0$ and all $z \in Q(t, y)$, $|z| \leqq 2 \sigma$ we have $\left|\operatorname{Inf}\left[z^{0} \mid\left(z^{0}, z\right) \in \widetilde{Q}(t, y)\right]\right| \leqq c$. The same holds for the sets $\widetilde{R}(t, \dot{y})$. Other alternate hypotheses have been discussed in [9]. Whenever the sets $\widetilde{Q}(t, y), \widetilde{R}(t, \dot{y})$ are not convex, existence theorems analogous to I and II below hold for generalized (strong, or weak) solutions.

A triple $(x, u, v), x \in S, u \in T, v \in \stackrel{\circ}{T}$, is said to be admissible provided relations (2), (3), (4), (5), (6), (7) hold, $f_{\mathrm{o}}(t,(\mathscr{M} x)(t), u(t))$ is $L$-integrable in $G$, and $g_{\circ}(t,(\mathscr{K} x)(t), v(t))$ is $\mu$-integrable on $\Gamma$.

EXISTENCE THEOREM $\underset{\widetilde{Q}}{\mathrm{I}}$ Under hypotheses $\left(\mathrm{H}_{1}\right),\left(\mathrm{H}_{2}\right),(\mathrm{C}),(\Psi)$, if for almost all $\bar{t} \in G$ the sets $\widetilde{\widetilde{Q}}(\bar{t}, y), y \in A(\bar{t})$, are convex and have property $(\mathrm{Q})$ with respect to $y$ in $A(\bar{t})$, if for $\mu$-almost all $\bar{t} \in \Gamma$ the sets $\widetilde{R}(\bar{t}, \dot{y}), \dot{y} \in B(\bar{t})$, are convex and have property $(\mathrm{Q})$ with respect to $\dot{y}$ in $B(t)$, if $\Omega$ is a nonempty closed class of admissible triples $(x, u, v)$ such that the set $\{x\}_{\Omega}$ is sequentially relatively compact in $(X, \tau)$, then the functional (1) attains its infimum in $\Omega$.

The proof is similar to the one in [8, statement (4.i)] with $\rho=r, \rho^{\prime}=r^{\prime}$, where now use is made of property $\left(\mathrm{K}_{w}\right)$ of the operators $\mathscr{L}$ and $\mathscr{J}$, and of lower closure theorems in [5, statement (7.i), condition $(\beta)$ of Remark 11], and corresponding extension to integrals defined on $\Gamma$ as in [8]. For further extensions of Existence Theorem I under alternate closure and convergence properties see P. Kaiser [12].

3. State equations in the weak form. We consider now the case where the functional equations (2), (3) (state equations) are written in the weak form, as it is customary in partial differential equation theory. To this purpose let $W$ denote a suitable normed space of test functions $w=$ $\left(w_{1}, w_{2}\right)$, where $w_{1} \in\left(L_{q}(G)\right)^{r}$ and $w_{2} \in\left(L_{q}(\Gamma)\right)^{r^{\prime}}$ are vector valued functions defined in $G$ and $\Gamma$ respectively, $p^{-1}+q^{-1}=1$ with the usual conventions. Let $W^{*}$ be the dual space of $W$.

We shall use the same general notations as in $\S 2$. Instead of the operators $\mathscr{L}$ and $\mathscr{J}$, we shall consider one operator $\mathscr{F}: S \rightarrow W^{*}$, so that $\mathscr{F} x$ for $x \in S$ is a bounded linear operator $\mathscr{F} x: W \rightarrow$ reals. For every $x \in S, u \in T, v \in \stackrel{\circ}{T}$ we consider also the operator $h_{x, u, v}$, or $h: W \rightarrow$ reals, 
defined by

$h_{x, u, v}\left(w_{1}, w_{2}\right)=\int_{G} f(t,(\mathscr{M} x)(t), u(t)) w_{1}(t) d t+\int_{\Gamma} g(t,(\mathscr{K} x)(t), v(t)) w_{2}(t) d \mu$

where $f w_{1}$ and $g w_{2}$ denote the usual inner products in $E^{r}$ and $E^{r^{\prime}}$ respectively. Instead of the state equations (2), (3) as before we consider now the unique state equation $\mathscr{F} x=h_{x, u, v}$, or explicitly $(\mathscr{F} x) w=h_{x, u, v}(w)$ for all $w \in W$, or

$$
(\mathfrak{5} x)\left(w_{1}, w_{2}\right)=h_{x, u, v}\left(w_{1}, w_{2}\right) \text { for all } w=\left(w_{1}, w_{2}\right) \in W .
$$

We are interested in finding elements $x \in S, u \in T, v \in \stackrel{\circ}{T}$, which minimize the functional (1) subject to the state equation (8) and the usual constraints (4), (5), (6), (7). A triple $(x, u, v)$ is now said to be admissible provided $x \in S, u \in T, v \in \stackrel{\circ}{T}$, relations (4), (5), (6), (7), (8) hold, the functions $f_{\mathrm{o}}(t,(\mathscr{M} x)(t), u(t)), f(t,(\mathscr{M} x)(t), u(t)), t \in G$, are of classes $L_{1}(G)$ and $\left(L_{1}(G)\right)^{r}$ respectively, and the functions $g_{\circ}(t,(\mathscr{K} x)(t), v(t))$, $g(t,(\mathscr{K} x)(t), v(t)), t \in \Gamma$, are of classes $L_{1}(\Gamma)$ and $\left(L_{1}(\Gamma)\right)^{r^{\prime}}$ respectively.

We shall say that the operator $\mathscr{F}: S \rightarrow W^{*}$ is weakly star closed with respect to $(X, \mathscr{S})$ (briefly, $\mathscr{F}$ has property $\mathrm{K}_{w}^{*}$ ) provided $x_{k} \in S$, $k=1,2, \cdots, x \in X, w^{*} \in W^{*}, x_{k} \rightarrow x$ in $(X, \tau)$, and $\left(\mathscr{F} x_{k}\right)(w) \rightarrow w^{*}(w)$ as $k \rightarrow \infty$ for every $w \in W$, implies $x \in \mathscr{S}$ and $\mathscr{F}(x)=w^{*}$. Instead of $\left(\mathbf{H}_{1}\right)$ we shall now require

$\left(\mathrm{H}_{1}^{*}\right)$ Hypotheses on $\mathscr{M}, \mathscr{K}, \mathscr{F}$. We shall assume that $\mathscr{M}: S \rightarrow\left(L_{1}(G)\right)^{\text {s }}$, $\mathscr{K}: S \rightarrow\left(L_{1}(\Gamma)\right)^{s^{\prime}}$ have the strong convergence property (property $\left.\mathrm{V}_{\sigma}\right)$, and that $\mathscr{F}: S \rightarrow W^{*}$ is weakly star closed (property $\mathrm{K}_{w}^{*}$ ) with respect to $(X, \tau)$.

$\left(\mathrm{H}_{3}\right)$ Hypotheses on $W, W^{*}$. We shall assume below that $\left(L_{p}(G)\right)^{r} \times$ $\left(L_{p}(\Gamma)\right)^{r^{\prime}} \subseteq W^{*}$, and that there is a constant $K>0$ such that

$$
\left\|w_{1}\right\|_{q}+\left\|w_{2}\right\|_{q}<K\|w\|_{W}
$$

where $\|w\|_{W}$ denotes the norm of $w=\left(w_{1}, w_{2}\right)$ in the normed space $W$.

$\left(\Psi_{p}\right)$ Growth hypotheses on $f_{\circ}, f$ and $g_{\circ}, g$. For $p=1$ we assume that $f_{\circ}, f, g_{\circ}, g$ satisfy growth condition ( $\Psi$ ) above. For $1<p<\infty$ we assume that there are functions $\Psi(t) \geqq 0, t \in G, \Psi \in L_{1}(G)$, and $\stackrel{\circ}{\Psi}^{\circ}(t) \geqq 0, t \in \Gamma$, $\stackrel{\circ}{\Psi} \in L_{1}(\Gamma)$, and constants $a>0, b>0$ such that

$$
\begin{aligned}
|f(t, y, u)|^{p} \leqq \Psi(t)+a f_{\circ}(t, y, u) & \text { for all }(t, y, u) \in M, \\
|g(t, \dot{y}, v)|^{p} \leqq \stackrel{\circ}{\Psi}(t)+b g_{\circ}(t, \dot{y}, v) & \text { for all }(t, \dot{y}, v) \in \stackrel{\circ}{M} .
\end{aligned}
$$

For $p=\infty$ we assume that there are functions $\Psi(t) \geqq 0, t \in G, \Psi \in L_{1}(G)$, $\stackrel{\odot}{\Psi}^{\circ}(t) \geqq 0, t \in \Gamma, \stackrel{\leftrightarrow}{\Psi} \in L_{1}(\Gamma)$ and a constant $c \geqq 0$ such that

$$
\begin{aligned}
& f_{\mathrm{o}}(t, y, u) \geqq-\Psi^{(}(t), \quad|f(t, y, u)| \leqq c \quad \text { for all }(t, y, u) \in M, \\
& g_{\circ}(t, \dot{y}, v) \geqq-\stackrel{\circ}{\Psi}(t), \quad|g(t, \dot{y}, v)| \leqq c \quad \text { for all }(t, \dot{y}, v) \in \stackrel{\circ}{M} \text {. }
\end{aligned}
$$


ExistenCe TheOREM II. Under hypotheses $\left(\mathrm{H}_{1}^{*}\right),\left(\mathrm{H}_{2}\right),\left(\mathrm{H}_{3}\right),\left(\Psi_{p}\right)$, $p \geqq 1$, if for almost every $\bar{t} \in G$ the sets $\widetilde{Q}(\bar{t}, y), y \in A(\bar{t})$, are convex and have property $(\mathrm{Q})$ with respect to $y$ in $A(\bar{t})$, if for $\mu$-almost all $\bar{t} \in \Gamma$ the sets $\widetilde{R}(\bar{t}, \stackrel{\circ}{y}), \stackrel{\circ}{y} \in B(\bar{t})$, are convex and have property $(\mathrm{Q})$ with respect to $\dot{y}$ in $B(\bar{t})$, if $\Omega$ is a nonempty closed class of admissible triples such that $\{x\}_{\Omega}$ is sequentially relatively compact in $(X, \tau)$, then the functional (1) attains its infimum in $\Omega$.

The proof is similar to the one in [5, statement (6.i)] where now use is made of property $\left(\mathrm{K}_{w}^{*}\right)$ of the operator $\mathscr{F}$. For further extensions of Existence Theorem II under alternate closure and convergence properties see P. Kaiser [12].

\section{REFERENCES}

1. L. Cesari, Existence theorems for problems of optimization with distributed and boundary controls, Proc. Internat. Congress of Math. (Nice, 1970), vol. 3, GauthierVillars, Paris, 1971, pp. 157-161.

2. - Existence theorems for weak and usual optimal solutions in Lagrange problems with unilateral constraints. I, II, Trans. Amer. Math. Soc. 124 (1966), 369412, 413-430. MR 34 \#3392; 3393.

3. — Existence theorems for optimal controls of the Mayer type, SIAM J. Control 6 (1968), 517-552. MR 39 \#4722.

4. - Seminormality and upper semicontinuity in optimal control, J. Optimization Theory Appl. 6 (1970), 114-137. MR 42 \#5139.

5. - Closure theorems for orientor fields and weak convergence, Arch. Rational Mech. Anal. (to appear).

6. - Lower semicontinuity and lower closure theorems without seminormality conditions, Ann. Mat. Pura Appl. 98 (1974), 381-397.

7. - A necessary and sufficient condition for lower semicontinuity, Bull. Amer. Math. Soc. 80 (1974), 467-472.

8. L. Cesari and D. E. Cowles, Existence theorems for optimization problems with distributed and boundary controls, Arch. Rational Mech. Anal. 46 (1972), 321-355.

9. L. Cesari and M. B. Suryanarayana, Closure theorems without seminormality conditions, J. Optimization Theory Appl. (to appear).

10. D. E. Cowles, Upper semicontinuity properties of variable sets in optimal control, J. Optimization Theory Appl. 10 (1972), 222-236.

11. N. Dunford and J. T. Schwartz, Linear operators. II. Spectral theory. Selfadjoint operators in Hilbert space, Interscience, New York, 1963. MR 32 \#6181.

12. $P$. Kaiser, Closed operators in problems of optimal control with distributed parameters (to appear).

13. C. Kuratowski, Les fonctions semi-continues dans l'espace des ensembles fermés, Fund. Math. 18 (1932), 148-166.

14. G. J. Minty, Monotone (nonlinear) operators in Hilbert space, Duke Math. J. 29 (1962), 341-346. MR 29 \#6319.

Department of Mathematics, University of Michigan, Ann Arbor, Michigan 48104

Current address (P. Kaiser): Department of Mathematics, Lewis University, Lockport, Illinois 60441 